\title{
REFLEXIÓN, FORMACIÓN INICIAL Y MODELO INTERCONECTADO DE CRECIMIENTO PROFESIONAL DOCENTE. UN ESTUDIO DE CASO
}

\author{
REFLEXÃO, FORMAÇÃO INICIAL E MODELO INTERLIGADO DE \\ CRESCIMENTO PROFISSIONAL DOCENTE. UM ESTUDO DE CASO
}

\begin{abstract}
REFLECTION, INITIAL TRAINING AND INTERCONNECTED MODEL OF TEACHING PROFESSIONAL GROWTH. A CASE STUDY
\end{abstract}

DOI: $10.22481 /$ rbba.v10i02.9507

Guillermo Cutrera

Universidad Nacional de Mar del Plata, Mar del Plata, Buenos Aires, Argentina ORCID: https://orcid.org/0000-0001-8452-4538

Dirección electrónica: guillecutrera@gmail.com

María Basilisa García Universidad Nacional de Mar del Plata, Mar del Plata, Buenos Aires, Argentina ORCID: https://orcid.org/0000-0002-4282-6957 Dirección electrónica: bachigarcia@gmail.com

\begin{abstract}
RESUMEN
Diferentes investigaciones han advertido sobre el impacto, comparativamente bajo, que presenta la formación inicial en el ejercicio profesional respecto de otras instancias formativas propias del desarrollo profesional docente. En este trabajo utilizamos el Modelo Interconectado de Crecimiento Profesional Docente (MICPD) para evidenciar cambios en Conocimiento Profesional de una futura profesora de química durante sus experiencias de aprendizaje en la materia Didáctica General y Especial, perteneciente al Profesorado en Química. Contextualizando la propuesta del MICPD para la investigación a partir de los Dominios propuestos, identificamos las principales vías que expresan cambios reconocidos por la estudiante durante


un trayecto de su formación inicial recuperando, desde dimensiones del Conocimiento Didáctico del Contenido (CDC), los cambios reconocidos por la estudiante. Los resultados encontrados permiten evidenciar modificaciones en algunos de los componentes del CDC de la estudiante según el tipo de dispositivo empleado. Finalmente discutimos los alcances de los resultados de este trabajo considerando el empleo del MICPD en un contexto formativo novedoso, con relación a los tradicionalmente utilizados en las investigaciones en esta línea.

Palabras clave: Formación docente inicial. Modelo Interconectado de Crecimiento Profesional Docente. Conocimiento Didáctico del Contenido.

\section{RESUMO}

Diferentes pesquisas alertam sobre o impacto comparativamente baixo do treinamento inicial em comparação com outras instâncias de treinamento típicas do desenvolvimento profissional de professores. Neste trabalho utilizamos o Modelo Interconectado de Crescimento Profissional do Professor (MICPD) para mostrar as mudanças no Conhecimento Profissional de uma futura professora de Química durante suas experiências de aprendizagem em Didática Geral e Especial, pertencente à Faculdade de Química. Contextualizando la propuesta del MICPD para la investigación a partir dos Dominios propuestos, identificamos as principais vias que expressam mudanças reconhecidas pela aluna durante um curso de seu tranamento inicial recuperando, desde dimensiones del Conocimiento Didáctico del Contenido (CDC), los cambios reconocidos por a estudante. Os resultados encontrados mostram modificações em alguns dos componentes do CDC da aluna de acordo com o tipo de dispositivo utilizado. Por fim, discute-se os escopos dos resultados deste trabalho considerando a utilização do MICPD em um novo contexto educacional, em relação aos tradicionalmente utilizados em pesquisas nesta linha.

Palavras-chave: Formação inicial de professores. Modelo interconectado de crescimento profissional docente. Reflexão do professor. Conhecimento Didático de Conteúdo. 


\begin{abstract}
Several studies have warned about the comparatively little impact that initial training has on professional activity in reference to other training instances related to teachers professional development. In this work, we used the Interconnected Model of Teacher Professional Growth (IMTPG) to evidence changes in a future Chemistry teacher professional knowledge during her learning experience at the General and Special Didactics subject, belonging to the Chemistry Teacher Carreer. Contextualizing the IMTPG research proposal from the proposed Domains, we identify the central instruments that illustrate changes that the student is able to recognize during a stage of her initial training, restoring those recognized changes from different Pedagogical Content Knowledge (PCK) aspects. The results obtained enable us to highlight modifications in some components of the student PCK according to the specific type of device used. In the end, we discussed the scope of the results obtained from this work, considering the use of the IMTPG within an innovative training context with regards to those methods that have been traditionally used in research carried out in this field.
\end{abstract}

Keywords: Initial teacher training. Interconnected Model of Teacher Professional Growth. Pedagogical Content Knowledge.

\title{
INTRODUCCIÓN
}

Una dimensión central en la formación docente, presente en la mayoría de las reformas curriculares de los profesorados, es el desarrollo de prácticas reflexivas que formen profesionales capaces de investigar su enseñanza y pensar críticamente sobre su trabajo (Doyle, 1990). En este sentido, Fenstermacher (1986) afirma que ser capaz de establecer las razones de la propia acción y actuar de acuerdo con las propias razones es esencial para la enseñanza autónoma y profesional. La clave para sostener el proceso de reflexión no solo implica tomar conciencia de que existe un problema, sino buscar una variedad de formas de entenderlo (LOUGHRAN, 2002), colocando en diálogo, durante el análisis, las experiencias en el aula, las creencias sobre esas experiencias y marcos teóricos referenciales. La capacidad de reflexionar críticamente sobre la práctica en el aula es considerada como una parte esencial del crecimiento profesional docente (JAWORSKI, 2006) y, especialmente importante, para los docentes 
noveles y durante la formación inicial (KAMINSKI, 2003). Estas últimas consideraciones se inscriben en concepciones sobre el conocimiento profesional docente desarrolladas durante las últimas décadas en la comunidad de investigadores en enseñanza de las ciencias (PÉREZ GÓMEZ, 2010). Por un lado, estos consensos se expresan en asumir a la práctica como un escenario complejo, incierto y cambiante donde se producen interacciones que merecen la pena observar, contrastar, cuestionar y reformular ya que son espacios y procesos generadores de nuevos conocimientos (SCHÖN, 1998). Además, se sabe que las teorías personales de los docentes que operan en la práctica tienen cierto carácter implícito y que cualquier intento de hacerlas explícitas, verbalizables, implica redescribirlas a través de procesos de explicitación (POZO, 2001) que requieren del contraste y la reflexión respecto de su eficacia, sentido y utilidad. La formación, entonces, debe orientarse a develar lo implícito, y para ello, debe ser autoanalítica, reflexiva y crítica (RODRIGUEZ PECH, AMALILLA MOREJÓN, 2018). Sobre la base de estos acuerdos, asumimos que la interacción permanente entre la práctica docente y la teoría, acompañada de la reflexión que permita la autorregulación del aprendizaje, conforman un punto de partida indispensable para diseñar programas de formación docente que promuevan el desarrollo profesional.

Hasta el momento se han proyectado diferentes líneas de investigación que abordan el problema y que han permitido avanzar en propuestas superadoras de modelos tradicionales (BENAVIDES y LÓPEZ, 2020). No obstante, y asumiendo la relevancia de los aportes realizados por cada una de ellas, estimamos que es necesario profundizar en estudios que permitan evidenciar el crecimiento del conocimiento profesional a partir de prácticas reflexivas (VALLIANT, 2007), en particular, en el contexto de la formación docente inicial. A su vez, consideramos necesario avanzar en una investigación que, paralelamente al crecimiento profesional, desarrolle un modelo de seguimiento y evaluación del impacto durante la formación docente inicial que contribuya a estimar el valor real de determinadas estrategias de formación y el impacto que tienen en el profesorado (BENAVIDES y LÓPEZ, 2020). El presente trabajo se enmarca en una investigación más amplia centrada en evidenciar instancias de desarrollo en el conocimiento de los futuros profesores de ciencias exactas y experimentales y constituye un aporte en este sentido, en tanto presenta una propuesta de formación acompañada de una estrategia metodológica para su análisis evaluando los resultados a partir de un estudio de casos que aborda estas dos cuestiones en una futura profesora de química.

Revista RBBA $\mid$ Revista Binacional Brasil Argentina Vol. 10, num. 2, dez/2021, p. 322-346 


\section{REFLEXIÓN, FORMACIÓN INICIAL Y MODELO INTERCONECTADO DE CRECIMIENTO PROFESIONAL DOCENTE. UN ESTUDIO DE CASO}

\section{1. ¿Conocimiento profesional docente y desarrollo profesional?}

Las conceptualizaciones sobre el desarrollo profesional de los docentes, lo consideran como un proceso individual o colectivo, en el que operan experiencias de diversa naturaleza. En este sentido, la definición que aporta Day (2005) involucra las experiencias de aprendizaje surgidas tanto en un ambiente natural como aquellas que planificadas por instituciones o por los propios profesores. Para este autor:

es el proceso por el cual, solo y con otros, el profesorado revisa, renueva y extiende su compromiso como agente de cambio [...] y por el que adquiere y desarrolla críticamente los conocimientos, destrezas e inteligencia emocional esenciales para la reflexión, la planificación y la práctica profesional adecuadas [... . en cada fase de su vida docente" (DAY, 2005, p. 17).

La historia de vida del profesor contiene elementos entrelazados de cada categoría, y que contribuyen - o no- a su desarrollo profesional. Day (1998, p. 33) expresa que es importante definir el desarrollo profesional como multidimensional, como interacción dinámica entre las diferentes etapas del profesorado en las que caben sus experiencias biográficas, factores ambientales, carrera profesional, vida y fases de aprendizaje a lo largo de la vida.

La noción de desarrollo profesional ha sido relacionada a una serie de etapas iniciadas con el ingreso en la carrera profesional (SIKES, 1992; DAY, 2005). La formación inicial representa la posibilidad de una instancia formativa dotada de una potencialidad significativa para promover el DPD. Sin embargo, este impacto sobre el desarrollo profesional no debería suponer a esta instancia como un trayecto inicial "autosuficiente", sino a "ayudar a los futuros enseñantes a que interioricen durante su formación inicial las disposiciones y destrezas necesarias para estudiar su ejercicio docente y perfeccionarlo en el transcurso del tiempo, y el compromiso de responsabilizarse de su propio desarrollo profesional" (ZEICHNER, 1995: 386).

En este contexto, el DPD se vincula con la necesidad de definir qué tipo de conocimientos deberían desarrollar los profesores. En este sentido, el trabajo de Shulman (1987) proporciona una base sólida para aprender sobre el tipo de conocimiento que los profesores necesitan para enseñar y cómo transforman el contenido o el conocimiento curricular en conocimiento enseñable. Los fundamentos filosóficos y teóricos en los que, actualmente, se basan los movimientos de reformas de la educación científica son una consecuencia de la teoría del aprendizaje constructivista (LUERA y OTTO, 2005). Desde esta perspectiva, el aprendizaje

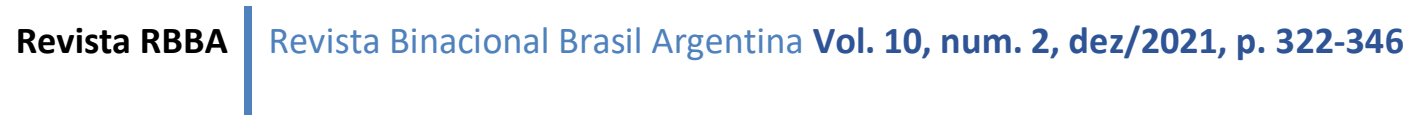


acontece sólo cuando los estudiantes construyen significados activamente a través de procesos personales y sociales e integran nuevos conocimientos en sus modelos mentales preexistentes del mundo (LUERA y OTTO, 2005). En este contexto, los profesores deben desarrollar formas especiales de conocimiento que les permitan transformar el conocimiento del contenido científico en formas que sean pedagógicamente poderosas y, sin embargo, adaptables a las variaciones en el conocimiento previo, los niveles de comprensión y las dificultades de aprendizaje que presentan los estudiantes. Esto es, precisamente, lo que Shulman (1986) conceptualiza como conocimiento pedagógico del contenido (PCK), en español, conocimiento didáctico del contenido (CDC). Asumimos al conocimiento profesional como un conjunto de conocimiento, creencias y acciones o actitudes, relacionado con los diferentes componentes del PCK que incluye la comprensión de los profesores de cómo los estudiantes aprenden, o no aprenden, una materia específica. Siguiendo la propuesta de Oliver y Parker (2008), centrada en el conjunto interconectado de seis dominios del conocimiento del profesor a través de los cuales se construye el PCK, recuperamos la centralidad de los procesos de reflexión en la acción o sobre la acción para la promoción de cambios en él y, a partir de ello, la consideración del profesor como un práctico reflexivo (SCHÖN, 1998).

\section{Un modelo para el seguimiento del desarrollo profesional}

Los campos de la formación inicial ofrecen la posibilidad de interacción del Conocimiento Profesional de los futuros profesores, conformado por conocimientos, creencias $\mathrm{y}$ actitudes en diferentes instancias formativas. Para poder seguir y estudiar el modo en que se va desarrollando este conocimiento profesional, recuperamos el modelo interconectado de crecimiento profesional (ICMPG ${ }^{\mathrm{i}}$, MICPD, en castellano) (CLARKE y HOLLINGSWORTH, 2002) que ofrece una heurística para la interpretación de los cambios que se van sucediendo. Este modelo sugiere que el cambio en el Conocimiento Profesional ocurre en ciclos recurrentes a través de los procesos mediadores de "reflexión" y "promulgación" en cuatro dominios distintos que abarcan el mundo del docente (CLARKE y HOLLINGSWORTH, 2002) (Figura 1): el dominio personal (DPe), el dominio de la práctica (DP), el dominio de las consecuencias (DC) y el dominio externo (DE). El dominio personal constituye el conocimiento, las actitudes y las creencias del profesor. El cambio en este dominio ocurre cuando los profesores adquieren nuevos conocimientos, actitudes o creencias. El dominio de la práctica se refiere a todas las

Revista RBBA $\mid$ Revista Binacional Brasil Argentina Vol. 10, num. 2, dez/2021, p. 322-346 
formas de experimentación profesional. Aunque Clarke y Hollingsworth (2002) señalan que el dominio de la práctica a menudo se limita a la experiencia de los profesores en el aula, mencionan explícitamente que la experimentación profesional de los profesores no se restringe únicamente a ella. El dominio de las consecuencias se ocupa de los resultados de las nuevas prácticas para los propios profesores y sus estudiantes. El cambio en este dominio se produce cuando los profesores perciben estos resultados como destacados. Los tres dominios forman parte de la vida profesional del docente y son denominados "dominios relacionados con los profesores" (Hilton, et. al., 2015). Uno de los dominios se encuentra fuera del mundo profesional del docente: el dominio externo. Este dominio ofrece al docente fuentes de información y/o estímulos y apoyo para desarrollar nuevas prácticas (WONGSOPAWIRO, 2012).

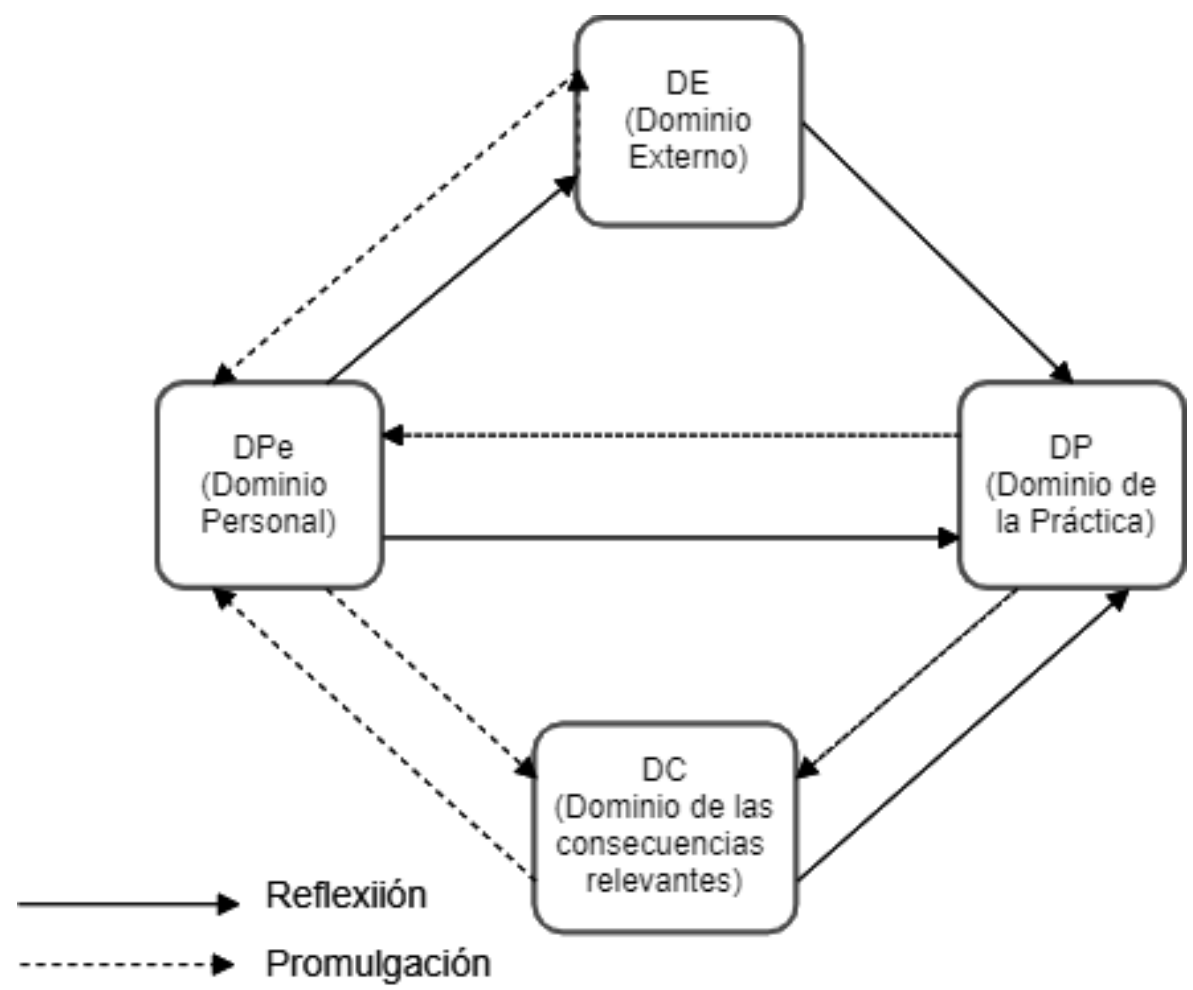

Figura 1. Dominios y tipos de mediación en el ICMPG. Fuente: Adaptado de Clarke, D. \& Hollingsworth, H. (2002). 
El modelo IMTPG nos recuerda que la formación del profesorado consiste en un proceso de aprendizaje profesional basado en la autorreflexión y la acción, de naturaleza no lineal, pudiendo darse de diversas formas en una variedad de contextos en línea con nuestra idea planteada en la introducción. También reconoce la complejidad del crecimiento profesional al identificar múltiples patrones de cambio y distinguir múltiples resultados del aprendizaje docente. La presencia de diferentes dominios a partir de los cuales, por medio de mediaciones, puede interpretarse los cambios en el conocimiento profesional permiten, para este trabajo, recuperar la diversidad de instancias formativas a partir de las cuales los futuros profesores pueden complejizar su conocimiento. Estos dominios son privilegiados, diferencialmente, en cada uno de los campos formativos mencionados con anterioridad.

\section{Preguntas y objetivo de la investigación}

\subsection{Objetivo}

Indagar en qué medida el Modelo Interconectado de Crecimiento Profesional Docente (MICPD) permite evidenciar cambios en Conocimiento Profesional de una futura profesora de química durante un tramo de su trayectoria formativa inicial.

\subsection{Preguntas de investigación}

1. ¿Cuáles son las principales vías que expresan cambios reconocidos por la estudiante durante un trayecto de su formación?

2. ¿Cómo pueden interpretarse los cambios evidenciados en la estudiante en términos del $\mathrm{CDC}$ ?

\section{Contexto de la investigación}

Con el objetivo de ofrecer espacios que promuevan la reflexión entendida en los términos descritos, las nuevas propuestas para la formación docente universitaria diseñadas por la Facultad de Ciencias Exactas y Naturales de la UNMdP en Argentina, se organizan en diferentes campos de conocimiento recorridos transversalmente en los que la promoción del desarrollo de la capacidad reflexiva durante la interacción entre teoría y práctica educativa es la tarea constitutiva central. En tal sentido, los planes de estudio plantean la enseñanza para el

Revista RBBA $\mid$ Revista Binacional Brasil Argentina Vol. 10, num. 2, dez/2021, p. 322-346 
ejercicio de una "práctica reflexiva", proponiendo espacios para la experimentación a lo largo de la formación (MARCELO y VAILLANT, 2010) e implicando contextos formativos que promuevan prácticas reflexivas más allá de la residencia docente (GARCÍA y CUTRERA, 2020), exigiendo que en cada una de las asignaturas por las que transcurre la formación del futuro docente se instalen ámbitos desde los cuales sea posible generar instancias que evidencien explícitamente el trabajo reflexivo.

Entendemos que una propuesta formativa para el DPD implica procesos y actividades diseñadas para mejorar los conocimientos y concepciones de los profesores e interpelar su acción en el aula de modo que se promueva el aprendizaje de sus estudiantes (GUSKEY, 2003). En este contexto, el DPD exige, como ya lo planteamos, de una práctica reflexiva interiorizada durante la instancia de la formación docente inicial. Esta instancia incluye la etapa formativa previa al desempeño de la profesión docente, encaminada a favorecer y desarrollar saberes vinculados al conocimiento profesional de los profesores, con el fin de prepararlos y facilitarles la realización eficaz de su tarea. La formación docente inicial debe ofrecer, entonces, instancias tanto para la adquisición de conocimientos vinculados a saberes disciplinares pertenecientes a los diferentes campos de conocimiento que componen la formación del profesor, históricamente construidos y validados como vinculados a la reflexión sobre las prácticas profesionales. En función de lo planteado, una vez establecida la propuesta curricular que permita las diferentes instancias reflexivas (GARCÍA y CUTRERA, 2020), el desafío siguiente reside en diseñar estrategias de seguimiento de esas reflexiones que promuevan el desarrollo profesional.

\section{Método}

Desde un paradigma interpretativo (SCHUSTER, PUENTE, ANDRADA Y MAIZA, 2013), el proyecto marco dentro del cual se desarrolló el presente trabajo busca identificar y describir los procesos de cambios que reconocen los profesores en formación en algunas dimensiones del CDC a medida que avanzaban en la carrera. El estudio involucra a estudiantes que están recorriendo el trayecto de la formación pedagógica y el de la práctica en la Facultad. En esta oportunidad presentamos los resultados correspondientes al caso representado por una estudiante durante la cursada en la asignatura "Didáctica de la Química", correspondiente al cuarto año de la carrera del Profesorado de Química. La estudiante, previamente había cursado todas las asignaturas que pertenecen al campo de la formación pedagógica y la mayor parte de las pertenecientes al campo disciplinar.

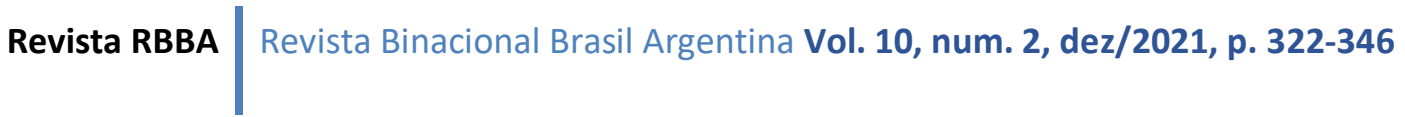


CRECIMIENTO PROFESIONAL DOCENTE. UN ESTUDIO DE CASO

\subsection{Propuesta de formación}

La propuesta de formación se diseñó en el contexto de un cambio de plan de estudios para los profesorados (GARCÍA y CUTRERA, 2020). Dicho cambio tuvo como eje central pensar la formación desde la reflexión en y sobre la acción (SCHÖN, 1998) lo que requirió incorporar obligaciones curriculares vinculadas a la práctica desde los primeros años de la carrera creando, para esto, talleres asociados a diferentes asignaturas. ${ }^{\text {ii }}$

La idea fundamental de la propuesta de formación reside en diseñar contextos formativos que permitan la reflexión sobre la propia acción en los diferentes talleres de práctica que propone el plan de estudios. Se busca generar estímulos de manera tal que los futuros docentes vayan construyendo su conocimiento profesional sobre la base de explicitar sus propias concepciones, poniéndolas en diálogo permanente con los nuevos conocimientos que le va proporcionando la carrera y con la experiencia práctica. En este sentido, las producciones de los estudiantes se asumen como objeto de investigación, reescribiendo el papel de las experiencias de la práctica en la formación profesional docente. En el caso particular de la asignatura Didáctica de la Química, objeto de estudio de este trabajo, la propuesta se concretó realizando un ciclo reflexivo en cada una de las unidades temáticas que la conforman y que se corresponden con algunos componentes del CDC (PARK, 2008; PARK, 2019): (a) finalidades y modelos de enseñanza, componentes de la dimensión correspondiente a las orientaciones para la enseñanza; (b) currículum; (c) recursos y estrategias de enseñanza y (d) evaluación.

Para cada uno de esos componentes se pensaron dispositivos que permitieron cumplir con un ciclo reflexivo que diseñamos de la siguiente manera: Inicio, etapa de explicitación de ideas previas, etapa de reestructuración reflexiva y etapa reflexión metacognitiva.

Inicio: se retoman tanto los resultados de las reflexiones como las dificultades explicitadas por los estudiantes en la clase anterior y se trabajan a partir del diseño de dispositivos específicos en función del tema. Puede ser dar una explicación, plantear un debate, realizar una tarea concreta.

Etapa de explicitación: en esta fase se utilizaron diarios de clase donde se diseñaron consignas tendientes a explicitar ideas respecto del tema en cuestión utilizando como disparador, en muchas oportunidades la propuesta inicial de clase elaborada por los estudiantes.

Revista RBBA $\mid$ Revista Binacional Brasil Argentina Vol. 10, num. 2, dez/2021, p. 322-346 


\section{REFLEXIÓN, FORMACIÓN INICIAL Y MODELO INTERCONECTADO DE CRECIMIENTO PROFESIONAL DOCENTE. UN ESTUDIO DE CASO}

Etapa de reestructuración reflexiva: durante esta etapa, según el tema a abordar, se implementaron distintas estrategias a través de dispositivos que permitían promover la reflexión: discusiones en foros, elaboración de actividades y posteriores reflexiones a partir de ellas en los diarios de clase, entrevistas, actividades en pequeños grupos, presentaciones individuales de sus propuestas de clase a los compañeros, pequeñas investigaciones, Representaciones del Contenido ( $\mathrm{ReCo}$ ). Estas estrategias fueron propuestas con la intención de acompañar los procesos de aprendizajes de los estudiantes, ofrecerles ayuda pedagógica y promover la toma de conciencia de sus propios procesos de aprendizaje.

Etapa de reestructuración metacognitiva: se trabajó con portafolios con consignas pensadas para que desencadenen procesos de autorregulación de los aprendizajes en sus percepciones y acciones docentes como, por ejemplo:

"Elegir entre 3 actividades/momentos que, desde tu opinión, representaron los aprendizajes más importantes y responder las siguientes consignas.

1. Aprendizajes logrados

2. Justificación de la elección

3. Sustento teórico, recuperando alguno de los textos de los trabajos prácticos

4. Reflexión crítica de la evidencia

5. Dificultades presentadas en la elaboración

6. Aspectos que se podrían mejorar"

Desde el inicio se trabajó con una propuesta de unidad didáctica sobre un tema en particular que los estudiantes elaboraron de manera individual al inicio de la cursada de la asignatura. De esta manera se buscó conocer sus concepciones y conocimientos iniciales sobre el tema a trabajar en el cuatrimestre y se fue trabajando con ellos y su propuesta didáctica a lo largo de la asignatura, mediante ciclos reflexivos planteados en cada tema abordado. La unidad didáctica presentada al inicio fue modificada a lo largo de toda la cursada en función de lo aprendido (Figura 2). 


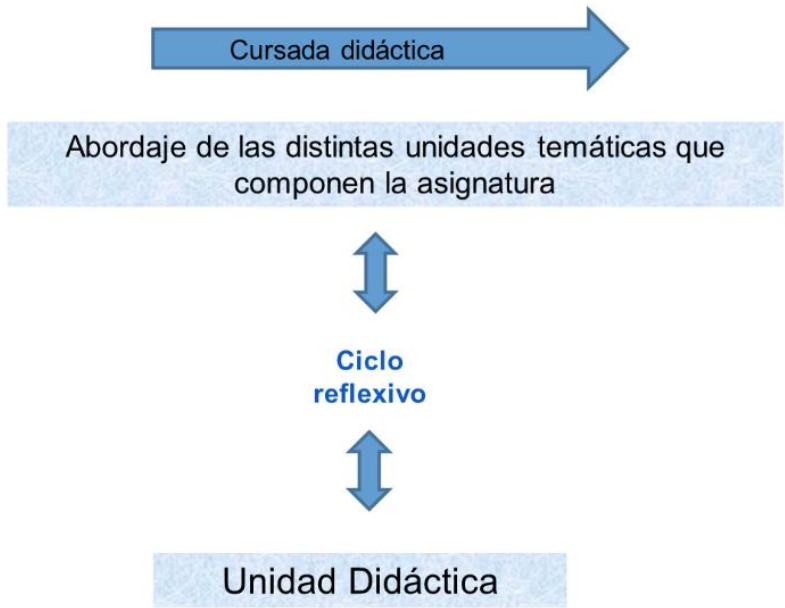

Figura 2. Inscripción de la unidad didáctica en el desarrollo de la materia. Fuente: elaboración propia.

La estrategia central para la formación se constituye, entonces, en un espacio de desarrollo del futuro profesor que reconoce su capacidad reflexiva sobre el conocimiento profesional.

6.2 Instrumentos: con relación a los instrumentos, las fuentes de datos utilizadas se corresponden con los dispositivos mencionados en la propuesta de formación.

6.3 Procedimiento de recolección y análisis de datos: Se llevaron a cabo las siguientes tareas:

6.3.1 Adaptación del Modelo: En función de las necesidades específicas de nuestro estudio, realizamos las siguientes adaptaciones del modelo.

En el dominio personal (DPe), lo consideramos como conjunto integrado de conocimientos teóricos y prácticos (cogniciones), creencias y actitudes. También incorporamos, en función de nuestro propósito de formación, las dificultades reconocidas por los estudiantes.

Dominio externo (DE): Dividimos el Dominio Externo subdominios en función de las instancias de participación ofrecidas en la propuesta de formación por medio de los dispositivos mencionados en la descripción de la propuesta.

Dominio de la Práctica: Recuperando la distinción propuesta por Zwart et al. (2007), para el DP en los subdominios denominados de preparación y enseñanza, en este trabajo circunscribimos al DP al subdominio de la preparación (DpP). 


\section{REFLEXIÓN, FORMACIÓN INICIAL Y MODELO INTERCONECTADO DE CRECIMIENTO PROFESIONAL DOCENTE. UN ESTUDIO DE CASO}

En este subdominio se consideraron los planes de clase que los participantes diseñaron para el aula y las clases aisladas en la etapa previa a la residencia junto con los documentos que reflejan las reflexiones realizadas a partir de la producción y la actuación. Se elaboraron indicadores para identificar las acciones que los estudiantes realizaron sobre su propuesta de clase.

Para estudiar cómo un cambio en un dominio desencadena un cambio en otro dominio, utilizamos los procesos mediadores de 'promulgación' y 'reflexión' que propone el modelo y los criterios adaptados por Justi y Van Driel (2006) (ver Tabla 1).

Representación de datos: La última adaptación realizada está vinculada a la representación que realizamos de los pictogramas cuando detectamos que un cambio en un dominio provocó cambios en dos diferentes dominios. En este caso, utilizamos dos flechas (igualmente numerados) para indicar procesos de mediación paralelos.

Por ejemplo, encontramos situaciones en las que la estudiante reconoce que una actividad realizada en el Dominio Externo provocó un cambio en su conocimiento (Dominio Personal) que posibilitó cambios en su Unidad Didáctica (Dominio de Práctica). En este caso, ambos cambios fueron identificados con el mismo número. Por ejemplo: "con la actividad que se nos planteó para describir los objetivos de cada anexo pude practicar, por lo que a la hora de corregir ciertos errores pude comprenderlos mejor y así retomar y repensar los objetivos de mi propuesta inicial (del DE al DPe y del DPe al DpP) (ver Figura 3)

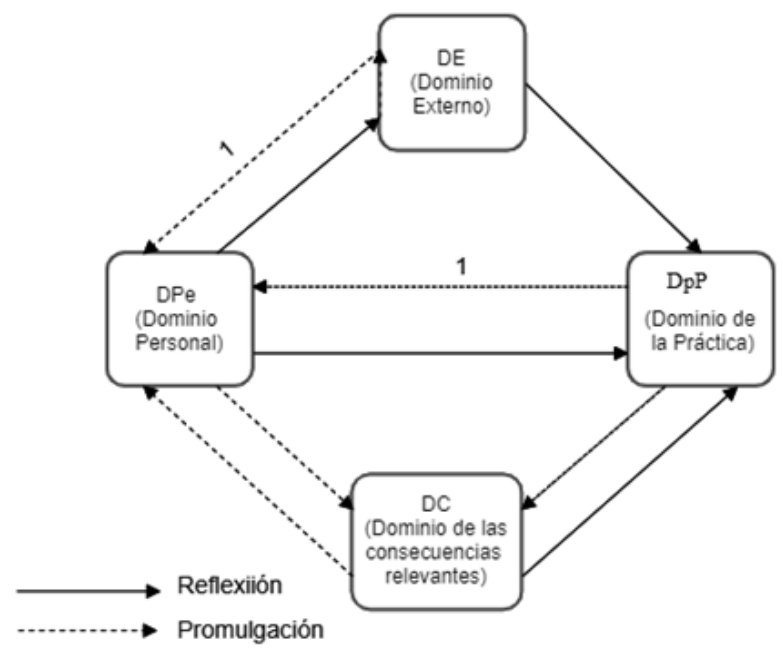

Figura 3. Cambios vinculantes entre diferentes dominios en una misma reflexión o promulgación. Fuente: Adaptado de CLARKE, D. \& HOLLINGSWORTH, H. (2002). 


\section{REFLEXIÓN, FORMACIÓN INICIAL Y MODELO INTERCONECTADO DE CRECIMIENTO PROFESIONAL DOCENTE. UN ESTUDIO DE CASO}

\subsubsection{Elaboración de indicadores}

A partir de los dominios de cambio que propone el modelo y los subdominios seleccionados para el análisis, se elaboraron indicadores que permitieron recolectar las expresiones que dieron cuenta de reflexiones y promulgaciones realizadas en los distintos momentos a través de todos los instrumentos utilizados. En la Tabla 1 se presentan, a modo de ejemplo, los indicadores relevantes en cada subdominio del dominio personal que es el que va dar cuenta de los cambios reconocidos o percibidos por la estudiante.

Tabla 1. Categorías relacionadas a los cambios reconocidos por la estudiante e indicadores asociados. Fuente: elaboración propia.

\begin{tabular}{|c|c|c|}
\hline \multicolumn{2}{|c|}{ Dominio de cambio } & Indicadores \\
\hline \multirow{4}{*}{$\begin{array}{l}\text { Conocimiento } \\
\text { Profesional }\end{array}$} & $\begin{array}{l}\text { Aprendizajes } \\
\text { reconocidos }\end{array}$ & $\begin{array}{l}\text { "No imaginaba que...., me } \\
\text { sorprendió que..." } \\
\text { "Me di cuenta que ...." } \\
\text { "Me faltó agregar...." } \\
\text { "Me permitió reconocer errores" } \\
\text { "Pude aprender" }\end{array}$ \\
\hline & Creencias modificadas & $\begin{array}{l}\text { "No los tenía en cuenta hasta que...." } \\
\text { "Mi concepción sobre ......era..." } \\
\text { "Nunca me había detenido a } \\
\text { pensar...." }\end{array}$ \\
\hline & Dificultades explicitadas & $\begin{array}{l}\text { "Creo que debería modificar..." } \\
\text { "Visualicé que tuve dificultades } \\
\text { en...." } \\
\text { "El objetivo pasó a ser.... y no } \\
\text { quedarme con...." }\end{array}$ \\
\hline & Acciones implementadas & $\begin{array}{l}\text { "Con lo que aprendi en......pude } \\
\text { incluir en mi propuesta......" } \\
\text { "Voy a modificar las actividades..." } \\
\text { "Pude adaptar actividades..." } \\
\text { "pude incluirla en mi propuesta } \\
\text { didáctica..." }\end{array}$ \\
\hline
\end{tabular}

\subsubsection{Identificación de promulgaciones y reflexiones}

Utilizando los criterios adaptados de Justi y Van Driel (2006) examinamos estos cambios buscando relaciones entre dominios del IMTPG, denominadas vías de cambio. Luego determinamos en qué dominios se produjo el cambio y lo codificamos. Por ejemplo: como resultado de una actividad planteada en el diario de clase, la estudiante dice: 
"Con los recursos brindados por los docentes para plantear actividades de indagación de ideas previas pude incluirla en mi propuesta didáctica; considero que el hecho de tener que convertir la actividad a una plataforma online para que estudiantes la realicen, me permitió aprender también sobre la creación de formularios, que me parece una herramienta de mucha utilidad para que el alumno interactúe permanentemente y de manera fácil con el docente." (P del DE al DP)

Como resultado de una participación en un foro en el que tenían que ver el trabajo de otro compañero y realizar aportes productivos, la estudiante expresa:

\begin{abstract}
"El hecho de realizar un comentario luego de ver los videos de otros compañeros me resultó interesante porque me permitió visualizar otras maneras de expresar la propuesta, conocer otras formas de plantear las actividades y reconocer errores en mi presentación y propuesta didáctica." (P del DE al DP)
\end{abstract}

\title{
6.3.4 Caracterización de vías de cambio
}

Una vez identificados todas las relaciones construimos los pictogramas correspondientes. Con el objetivo de caracterizar los cambios, analizamos los pictogramas desde los dominios del modelo interconectado y a partir de las categorías de estudio para las que elaboramos indicadores.

\subsubsection{Análisis de los cambios desde la perspectiva del CDC}

Por último, clasificamos los cambios reconocidos por la estudiante según los componentes del CDC descritos por Park y Oliver (2008). Por último, retomamos las categorías profesionales estudiadas a partir del MICPD desde los componentes del CDC para ver en qué medida se pudieron promover cambios y cómo pueden ser descritos.

\section{Resultados}

\subsection{Caracterización de los cambios reconocidos por la estudiante}

Con relación a las instancias de mediación entre dominios manifestadas por la estudiante, se presentaron frecuencias de codificación mayores para la reflexión $(n=32)$ respecto de la promulgación $(n=8)$.

Revista RBBA $\mid$ Revista Binacional Brasil Argentina Vol. 10, num. 2, dez/2021, p. 322-346 


\section{REFLEXIÓN, FORMACIÓN INICIAL Y MODELO INTERCONECTADO DE CRECIMIENTO PROFESIONAL DOCENTE. UN ESTUDIO DE CASO}

Las vías de cambio evidenciadas en estas mediaciones se presentaron entre los dominios personal y externo $(n=33)$, los dominios externos y de la práctica $(n=10)$ y entre los dominios personal y de la práctica (n=1) (Figura 4).

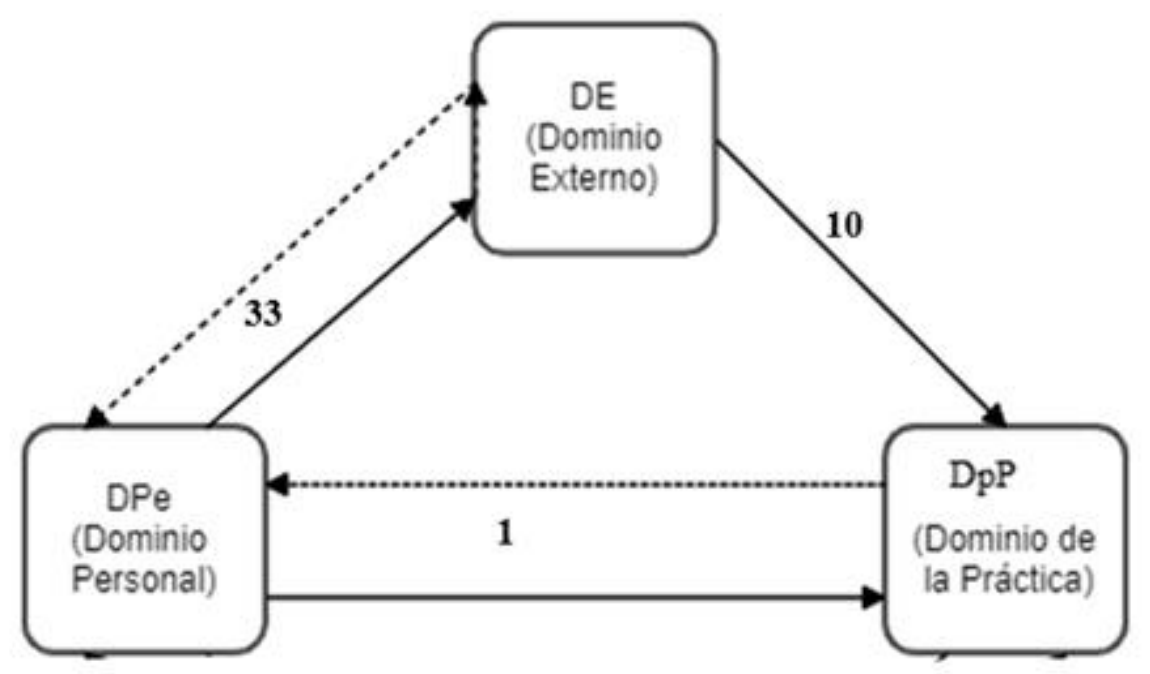

Figura 4. Frecuencias totales para las mediaciones entre dominios. Fuente: elaboración propia.

Los cambios registrados por la estudiante fueron categorizados recuperando las palabras utilizadas por ella en sus promulgaciones y reflexiones. Estos cambios se expresaron en términos de aprendizajes reconocidos al realizar trabajos prácticos de la asignatura, creencias modificadas y dificultades explicitadas, generalmente volcadas en los diarios de clases, y modificaciones que, progresivamente, pudo realizar a su propuesta didáctica. Si bien estas últimas pueden ser consideradas inclusivas de las anteriores, no obstante, decidimos considerarlas como una categoría diferencial, respetando la referencia explícita al término "aprendizaje" por la estudiante en sus textos. De este conjunto de categorías vinculadas al cambio, las de mayor frecuencia correspondieron al reconocimiento de aprendizajes $(n=22)$ y a los aportes a su propuesta didáctica $(\mathrm{n}=10)$. Las referidas a creencias iniciales modificadas por la estudiante durante el trabajo en la materia y las dificultades explicitadas, presentaron una menor frecuencia de codificación ( $\mathrm{n}=3$, en cada una de las categorías).

En la figura 5 se presentan y ejemplifican las categorías correspondientes a los cambios reconocidos por la propia estudiante en su conocimiento. 


\section{REFLEXIÓN, FORMACIÓN INICIAL Y MODELO INTERCONECTADO DE CRECIMIENTO PROFESIONAL DOCENTE. UN ESTUDIO DE CASO}

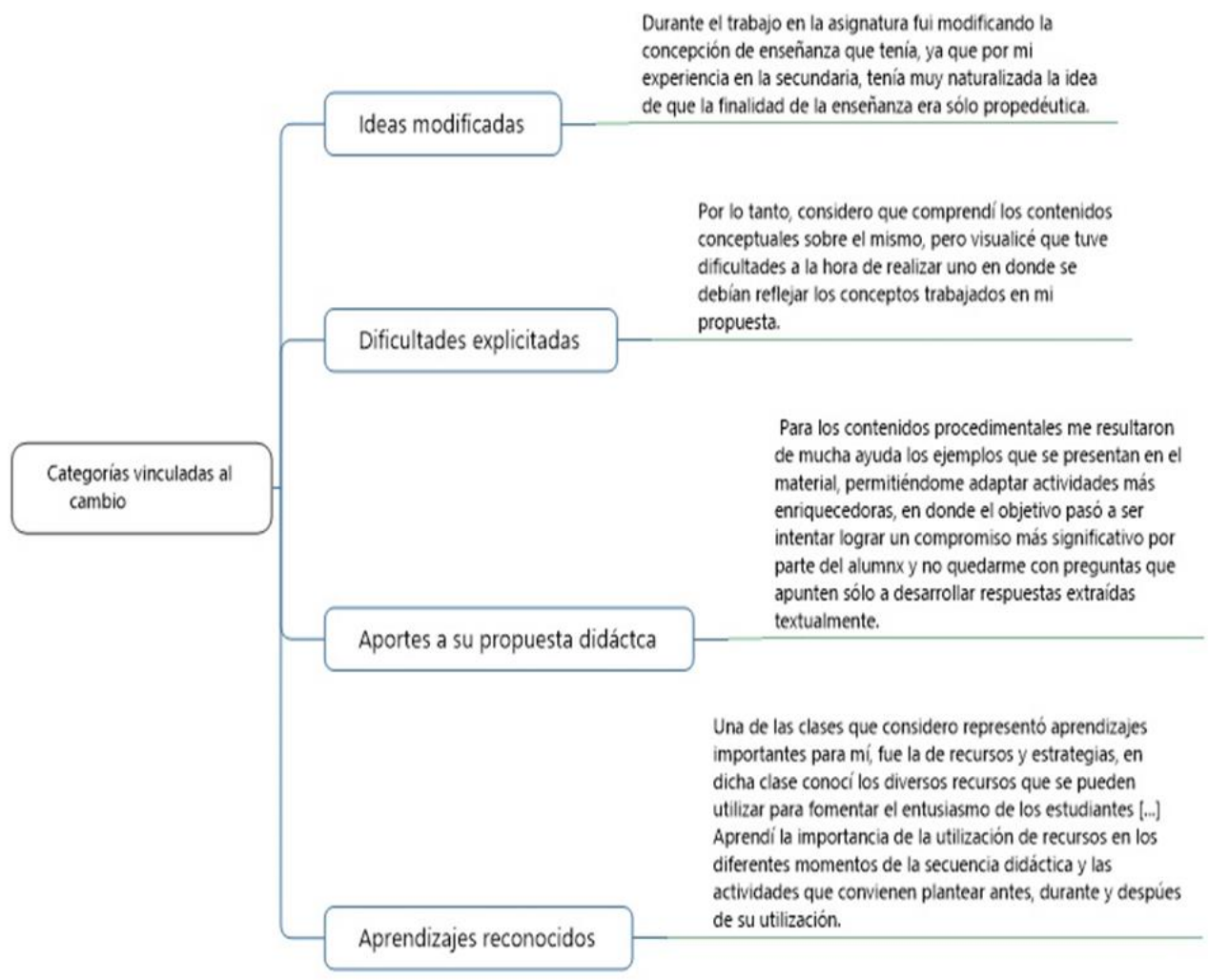

Figura 5. Categorías asociadas al cambio en algunas dimensiones en el conocimiento de la estudiante. Fuente: elaboración propia.

Entre los aportes recuperados por la estudiantes a partir de las actividades en las que se involucró, podemos destacar los referidos a la formulación de objetivos ("Pero, con la actividad que se nos planteó para describir los objetivos de cada anexo pude practicar, por lo que a la hora de corregir ciertos errores pude comprenderlos mejor y así retomar y repensar los objetivos de mi propuesta inicial"); selección de estrategias ("Considero que analizar las estrategias y recursos que se encuentran disponibles para aplicar en el aula, fue un aprendizaje muy enriquecedor, ya que a partir de ese momento, comenzaron a surgir las ideas sobre cómo poder mejorar los contenidos de mi propuesta de acuerdo a mis finalidades") y de contenidos procedimentales ("Considero que analizar las estrategias y recursos que se encuentran disponibles para aplicar en el aula, fue un aprendizaje muy enriquecedor, ya que a partir de ese momento, comenzaron a surgir las ideas sobre cómo poder mejorar los contenidos de mi propuesta de acuerdo a mis finalidades"); indagación de saberes previos ("Considero que analizar las estrategias y recursos que se encuentran disponibles para aplicar en el aula, fue un aprendizaje muy enriquecedor, ya que a partir de ese momento, comenzaron a surgir las 


\section{REFLEXIÓN, FORMACIÓN INICIAL Y MODELO INTERCONECTADO DE CRECIMIENTO PROFESIONAL DOCENTE. UN ESTUDIO DE CASO}

ideas sobre cómo poder mejorar los contenidos de mi propuesta de acuerdo a mis finalidades"); reformulación de temáticas ("La lectura del texto y los ejemplos que se brindaron en el mismo me permitieron comprender el concepto y lo más importante de todo, fue que pude darme cuenta que en mi propuesta faltaban actividades de carácter social") y de objetivos ("Caí en la cuenta que mi finalidad de enseñanza, claramente, no era compatible con los objetivos y contenidos que había planteado para la propuesta, la primera vez que la redacté"); uso de recursos ("Luego de la clase sobre la utilización de las TICs me di cuenta que no incorporé muchas de ellas y que a su vez, para el video propuesto me faltó agregar actividades para antes, durante y después del mismo, por lo que claramente debo modificarlo"); textos como recursos didácticos ("Considero que analizar las estrategias y recursos que se encuentran disponibles para aplicar en el aula, fue un aprendizaje muy enriquecedor, ya que a partir de ese momento, comenzaron a surgir las ideas sobre cómo poder mejorar los contenidos de mi propuesta de acuerdo a mis finalidades").

\subsection{Análisis de los cambios desde la perspectiva del CDC}

El contenido de los cambios reconocidos por la estudiante puede ser leído, a su vez, en términos de diferentes dimensiones del CDC. Para esto, las citas encontradas fueron agrupadas en las siguientes dimensiones que constituyen parte de los componentes que describen Park y Oliver (2008) para el CDC: modelos de enseñanza, recursos y estrategias de enseñanza, evaluación y diseño curricular (Figura 6).

Las referencias a recursos y estrategias de enseñanza fueron las que presentaron mayor frecuencia de codificación $(n=21)$. En esta dimensión se incluyen valoraciones de la estudiante respecto del empleo de la historia de la ciencia ( "En la actividad 1 del trabajo pude comprender la importancia de incorporar textos con historia en las clases de ciencias, ya que por ejemplo, la participación en el foro me permitió reflexionar sobre muchas situaciones que se vivieron en diferentes contextos a lo largo de la historia”); al empleo de textos (“[...] pude interpretar la importancia del uso de textos las clases de ciencias, ya que el objetivo principal es que lxs alumnxs desarrollen la capacidad de establecer relaciones entre los conceptos que se expresan en los textos y los conocimientos adquiridos en otras situaciones"); relacionadas al empleo de mapas conceptuales (“[..] si bien tenía las ideas principales de cómo crear uno, me sorprendió la amplia gama de posibilidades que hay para poder llevarlo al aula [...]”); empleo de TIC ("Considero que analizar las estrategias y recursos que se encuentran disponibles para aplicar

Revista RBBA $\mid$ Revista Binacional Brasil Argentina Vol. 10, num. 2, dez/2021, p. 322-346 


\section{REFLEXIÓN, FORMACIÓN INICIAL Y MODELO INTERCONECTADO DE CRECIMIENTO PROFESIONAL DOCENTE. UN ESTUDIO DE CASO}

en el aula, fue un aprendizaje muy enriquecedor, ya que a partir de ese momento, comenzaron a surgir las ideas sobre cómo poder mejorar los contenidos de mi propuesta de acuerdo a mis finalidades").

Por otra parte, la estudiante refirió a aspectos relacionados a la finalidad de la enseñanza $(\mathrm{n}=3)$ ("A su vez, mediante la lectura de los textos propuestos para el trabajo pude comprender muy bien la diferencia que radica en cada finalidad de enseñanza”). Estas citas, en tanto refieren a uno, entre otros aspectos que definen un modelo de enseñanza, se incluyeron en esta última dimensión del CDC. Las referencias a las dimensiones asociadas a la evaluación $(n=6)$ (“Algo importante a mencionar son los criterios de evaluación que no los tenía en cuenta hasta que se mencionaron”) y al diseño curricular tienen frecuencias similares. Con relación al a diseño curricular $(\mathrm{n}=11)$, la estudiante refiere en términos de la organización de los contenidos ("Destaco lo importante que fue conocer la existencia de ejes organizadores"); objetivos ("Destaco una mejor comprensión sobre la identificación y redacción de los objetivos de aprendizaje de una actividad, ya que lo veníamos trabajando anteriormente"); tipos de contenidos ("Para los contenidos procedimentales me resultaron de mucha ayuda los ejemplos que se presentan en el material”).

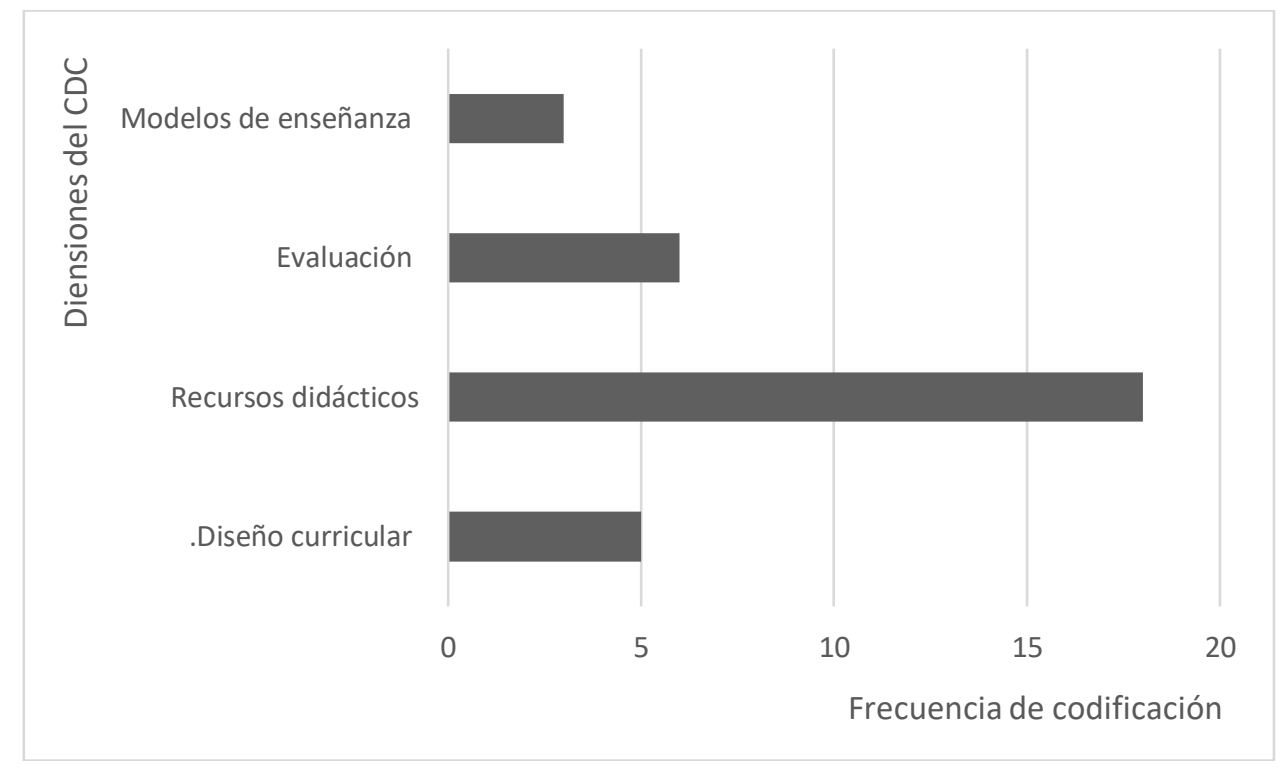

Figura 6. Componentes del CDC priorizadas en los textos elaborados por la estudiante. Fuente: elaboración propia.

Estas dimensiones del CDC se expresaron con frecuencias diferentes según el cambio entre dominios. La mayor frecuencia de codificación para los cambios del dominio externo al 


\section{REFLEXIÓN, FORMACIÓN INICIAL Y MODELO INTERCONECTADO DE CRECIMIENTO PROFESIONAL DOCENTE. UN ESTUDIO DE CASO}

personal, con relación a los restantes cambios, se expresa también en una mayor codificación en cada una de las dimensiones del CDC. Mientras que cada una de las dimensiones del CDC (diseño curricular, evaluación, modelos de enseñanza y recursos didácticos) se expresa en los cambios entre estos dominios (personal y externo), el cambio del dominio externo al de la práctica involucra las dimensiones correspondientes al diseño curricular, evaluación y recursos didácticos. En el caso del cambio entre el dominio personal al de la práctica, la única dimensión presente corresponde al diseño curricular. En la Figura 7 se presentan estas relaciones con las respectivas frecuencias.

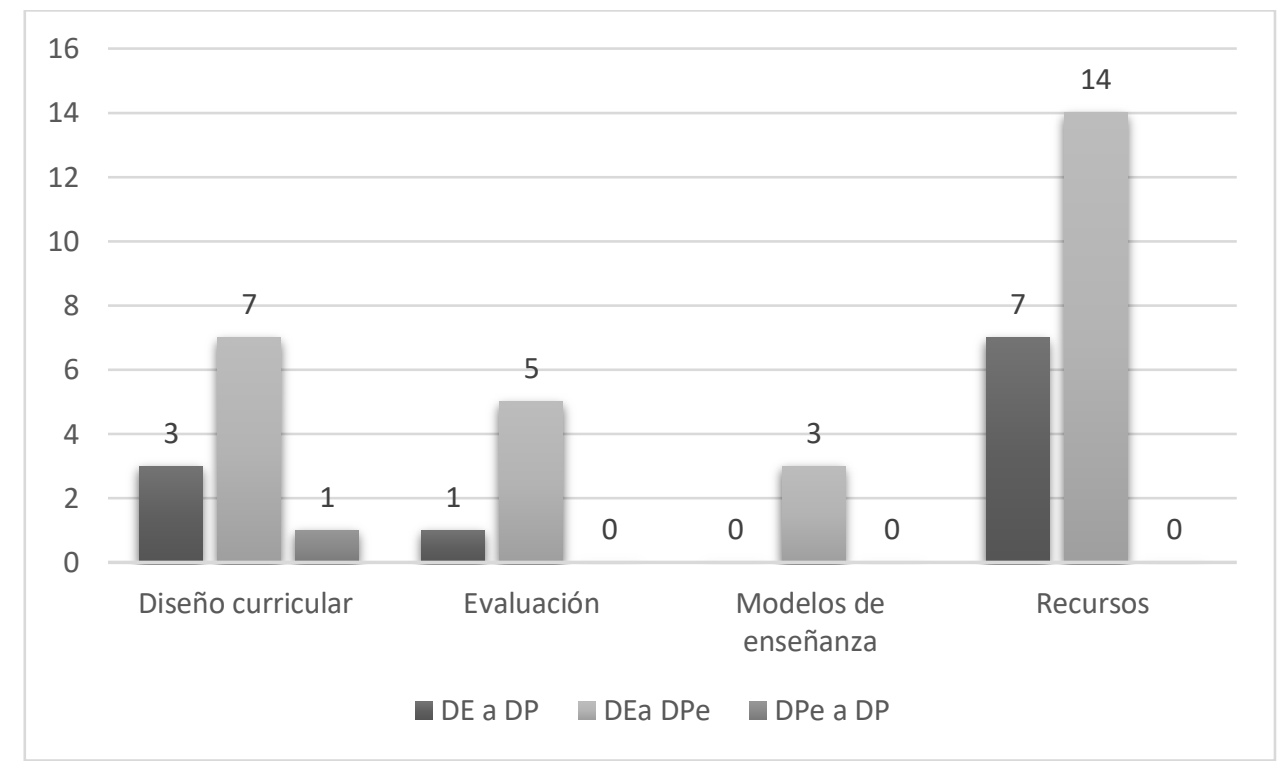

Figura 7. Frecuencias de codificación para las diferentes dimensiones del CDC según el tipo de cambio entre dominios. Fuente: elaboración propia.

Partiendo de la idea que el desarrollo profesional implica aumentar y complejizar los conocimientos, explicitando y, eventualmente, redescribriendo creencias que se reflejen en nuevas acciones, se leyeron los cambios desde los tres componentes del Conocimiento Profesional (conocimientos, creencias y acciones), de manera tal de evidenciar no sólo en qué dominios se produjeron los desarrollos sino, también, cuáles fueron sus características (Figura $8)$. 


\section{CRECIMIENTO PROFESIONAL DOCENTE. UN ESTUDIO DE CASO}

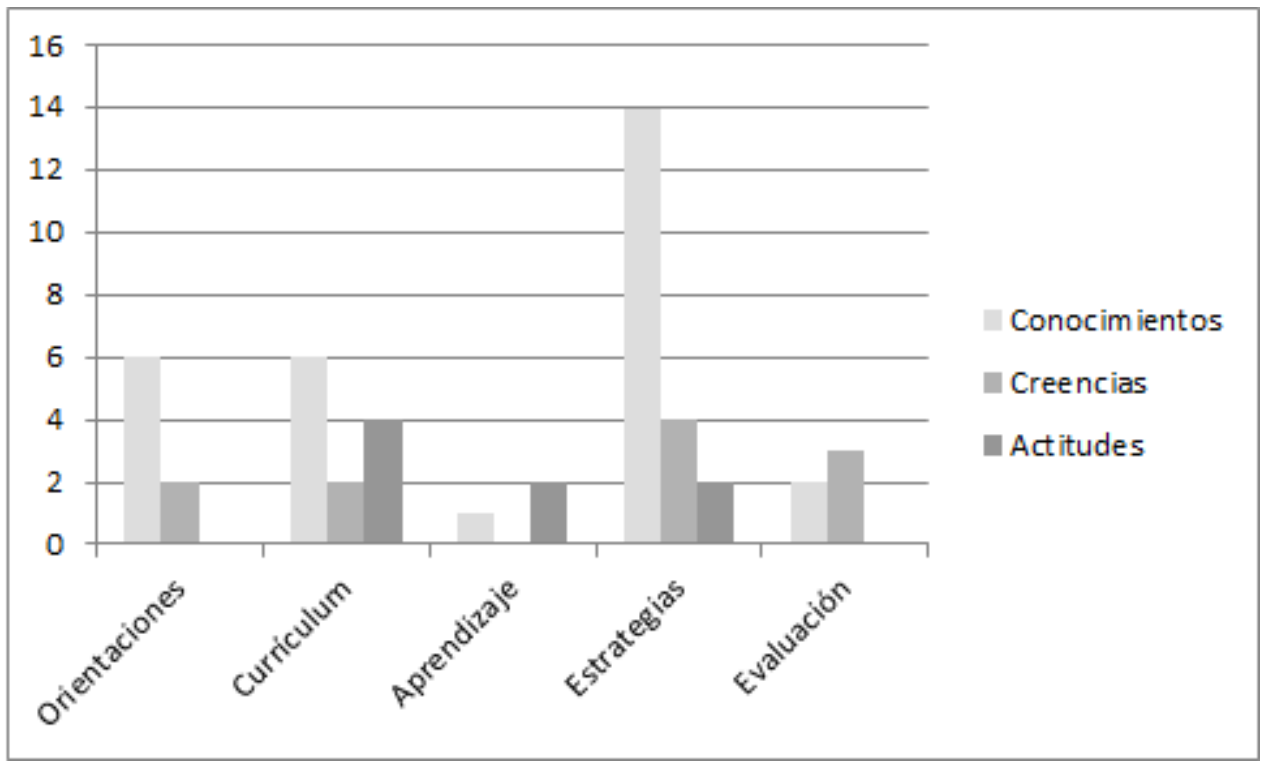

Figura 8. Frecuencias de codificación para componentes del CDC. Fuente: elaboración propia.

Teniendo en cuenta que la asignatura estudiada es didáctica, no resulta sorprendente que los cambios que más se han dado estén vinculados con el reconocimiento de nuevos aprendizajes por parte de la estudiante. Es esperable que, durante las siguientes instancias de este recorrido formativo inicial, se evidencien nuevos cambios en las creencias y acciones, en tanto componentes del CP. Tanto las creencias como las acciones, muchas veces están dirigidas por las representaciones con cierto carácter implícito, menos conscientes, y suelen resultar más difíciles de modificar dado que funcionan de manera más duradera, más robusta y con menor costo cognitivo (REBER, 1993) requiriendo de abundantes procesos de la reflexión sobre la práctica, que se da con mayor intensidad durante el período de residencia.

El reconocimiento por parte de los estudiantes de cambios en sus creencias aporta evidencia empírica para la postura sostenida por Richardson and Placier (2001) respecto de que algunas creencias pueden ser modificadas en instancias previas a la práctica, en oposición a la postura de (GUSKEY, 2002) que sostiene que los cambios significativos en las creencias y actitudes de los docentes tienen lugar solo después de que se hayan hecho evidentes cambios en los resultados de aprendizaje de los estudiantes, es decir, una vez que los docentes hayan "probado en el terreno" las propuestas de para el aula y hayan experimentado cambios de primera mano en los resultados de aprendizaje de los estudiantes (CLARKE \& HOLLINGSWORTH, 2002, p. 949). 
CRECIMIENTO PROFESIONAL DOCENTE. UN ESTUDIO DE CASO

\section{Conclusiones}

Los cambios en el conocimiento, dominio personal, reconocidos por la estudiante en el trayecto formativo ofrecido en la materia, se inscribieron en las relaciones con los dominios de la práctica y externo, es decir con los estímulos proporcionados desde la propuesta de formación. Si bien resultan previsibles en el contexto de las oportunidades formativas ofrecidas por la propuesta docente en la materia, permiten una lectura crítica de la propuesta de formación desde los cambios a partir de los dispositivos utilizados y de la preeminencia de unos cambios sobre otros. Por otra parte, la elaboración de la propuesta didáctica favoreció instancias reflexivas en la estudiante, presentándose como una vía relevante para recuperar contextualizadamente diferentes temáticas trabajadas en la materia. Si bien esta recuperación es previsible desde los propósitos de la materia, lo interesante es que ofreció un contexto formativo relevante, además vivenciado en estos términos por la estudiante. No obstante, los resultados obtenidos demandan una revisión de las consignas en pos de aumentar las oportunidades de reflexión en torno a las creencias y actitudes, como así también alrededor de algunas temáticas que fueron comparativamente menos abordadas junto con la necesidad de incorporar espacios de práctica. De esta manera, la propuesta de formación se va mejorando, a partir de un proceso cíclico e iterativo, donde los resultados son un insumo indispensable para las mejoras en cada ciclo. Recuperando lo oportunamente expuesto, y como reclaman diferentes investigaciones, proporcionar a los futuros profesores oportunidades para reflexionar sobre lo que hacen en el aula y por qué lo hacen (LOUGHRAN, MULHALL y BERRY, 2008), es una condición necesaria para favorecer el trabajo sobre el conocimiento del profesorado respecto de la enseñanza.

Los resultados evidenciaron también la importancia de la utilización de herramientas que ayuden a sistematizar las reflexiones de manera tal que facilite su interpretación. En este sentido, el MICPD es una heurística que permitió proporcionar una lectura del proceso de aprendizaje recurriendo a contextos que resultan novedosos para aquellos privilegiados por las investigaciones en esta línea. Entendemos que la diferenciación de dominios a través de los cuales pueden surgir líneas de cambio en el conocimiento profesional permite enriquecer las oportunidades para la formación de docentes en las prácticas reflexivas. Los resultados obtenidos en este aspecto ofrecen una lectura adicional en términos de la revisión de los dispositivos utilizados en la asignatura y las oportunidades de interacción con pares y docentes que permitan potenciar las relaciones del dominio personal con el dominio de la práctica.

\begin{tabular}{l|l} 
Revista RBBA & Revista Binacional Brasil Argentina Vol. 10, num. 2, dez/2021, p. 322-346
\end{tabular} 


\section{REFLEXIÓN, FORMACIÓN INICIAL Y MODELO INTERCONECTADO DE CRECIMIENTO PROFESIONAL DOCENTE. UN ESTUDIO DE CASO}

Actualmente, este contexto, como se indicará oportunamente, es circunscripto a la elaboración de unidades didácticas. No obstante, este dominio puede expandirse en un doble sentido. Por un lado, en intervenciones contextualizadas en instituciones escolares que permitan un primer acercamiento al análisis de prácticas de enseñanza de docentes en ejercicio, ampliando las posibilidades de vínculos entre ambos dominios (personal y de la práctica); por otra parte, en la continuidad de instancias formativas ofrecidas por las asignaturas siguientes del trayecto formativo inicial. Por otra parte, esto pone en evidencia la importancia de un ingreso temprano de los estudiantes a las instituciones escolares durante su formación inicial considerando que los cambios potenciales en el conocimiento profesional de los futuros profesores requieren de diferentes "puertas de ingreso". Para finalizar, se buscará expandir los resultados de esta investigación a otros casos, como aporte a la formación continua de docentes y al DPD.

\section{REFERENCIAS}

BENAVIDES, C. A. y LÓPEZ, N. M. Retos contemporáneos para la formación permanente del profesorado universitario. Educación $\mathbf{y}$ educadores, v. 23, n. 1, p. 71-88., 2020. https://doi.org/10.5294/edu.2020.23.1.4.

CAVANAGH, M., \& PRESCOTT, A. The growth of reflective practice among three beginning secondary mathematics teachers. Asia-Pacific Journal of Teacher Education, v. 38, n. 2, p. 147159, 2010. https://doi.org/10.1080/13598661003678968

CHIN, T. Y. (2015). Insights and lessons from primary science teachers' initial professional learning and collaborative practices in questioning. Narratives of Doctoral Studies. In: SIMON, S., OTTANDER, C., \& PARCHMANN, I. (Eds.). (2015). Narratives of Doctoral. Making the transition from educational practitioner to researcher. Cap. 14. Routledge., p. 166-178.

CLARKE, D., \& HOLLINGSWORTH, H. Elaborating a model of teacher professional growth. Teaching and Teacher Education, v. 18, n. 8, p. 947-967, 2002. https://doi.org/10.1016/S0742051X(02)00053-7

DAY, C. La formación permanente del profesorado en Europa: temas y condiciones para su desarrollo en el siglo XXI. Revista de educación, n. 317, p. 31-44, 1998.

DAY, C. Formar docentes: cómo, cuándo y en qué condiciones aprende el profesorado (Vol. 101). Madrid: Narcea Ediciones, 2005.

FENSTERMACHER, G. Philosophy of research on teaching: Three aspects. In WITTROCK, M. (Ed.) Handbook of Research on Teaching, p. 37-49. New York: Macmillan, 1986.

GARCÍA, MA. B.; CUTRERA, G. El campo de la práctica docente en la formación inicial. Un estudio descriptivo de una propuesta curricular para los profesorados de ciencias exactas y 
naturales. Revista RBBA-Revista Binacional Brasil Argentina: Diálogo entre as Ciencias. v. 9, n. 2, p. 176-196, 2020. https://doi.org/10.22481/rbba.v1i02.7805

GUSKEY, T. R. What makes professional development effective? Phi delta kappan, v. 84, n. 10, p. 748-750, 2003. https://doi.org/10.1177/003172170308401007

HOBAN, G. F. Making practice problematic: Listening to student interviews as a catalyst for teacher reflection. Asia-Pacific Journal of Teacher Education, 28(2), p. 133-147, 2000. https://doi.org/10.1080/713650685

JAWORSKI, B. Theory and practice in mathematics teaching development: Critical inquiry as a mode of learning in teaching. Journal of Mathematics Teacher Education, v. 9, n. 2, p. 187-2, 2006. https://doi.org/10.1016/S0742-051X(01)00051-8

JAY, J. K., \& JOHNSON, K. L. Capturing complexity: a typology of reflective practice for Education. Teaching and Teacher Education, 18, p. 73-85, 2002. https://doi.org/10.1016/S0742051X(01)00051-8

KAMINSKI, E. Promoting pre-service teacher education students' reflective practice in mathematics. Asia-Pacific Journal of Teacher Education, v. 31, n. 1, p. 21-32, 2003. https://doi.org/10.1080/13598660301619

LOUGHRAN, J., MULHALL, P., \& BERRY, A. Exploring pedagogical content knowledge in science teacher education. International Journal of Science Education, v. 30, n. 10, p. 1301-1320, 2008. https://doi.org/10.1080/1354060042000337110

MARCELO, C. \& VAILLANT, D. Desarrollo profesional docente: ¿cómo se aprende a enseñar? (Vol. 115). Madrid: Narcea Ediciones, 2010.

MARCELO, C. La evaluación del desarrollo profesional docente. En: VÉLAZ DE MEDRANO, C. Y VAILLANT, D. (Coords.). Aprendizaje y desarrollo profesional docente, p.119-127. Madrid: Funadcion Metas Educativas, 2011.

PARSONS, M., \& STEPHENSON, M. Developing reflective practice in student teachers: Collaboration and critical partnerships. Teachers and Teaching: theory and practice, v. 11, n. 1, p. $95-116,2005$. https://doi.org/10.1080/1354060042000337110

SCHÖN, D. El profesional reflexivo: cómo piensan los profesionales cuando actúan. Barcelona: Paidós, 1998.

PÉREZ GÓMEZ, A. El sentido del prácticum en la formación de docentes, La compleja interacción de la práctica y la teoría. In: BACHES MINOVES, N., CARBÓ TEIGEIRO, J. M., FERNÁNDEZ BERROCAL, P., BAUTISTA GARCÍA-VERA, A., HERNÁNDEZ ÁLVAREZ, A., MARRERO ACOSTA, J \& ZAFRA JIMÉNEZ, M.: Aprender a Enseñar la Práctica: Procesos de innovación y prácticas de formación en la Educación Secundaria. Barcelona: Edit. Graó, 2010.

PORLÁN ARIZA, R., MARTÍN DEL POZO, R., RIVERO GARCÍA, A., HARRES, J. B. S., AZCÁRATE GODED, M. D. P., \& PIZZATO, M. El cambio del profesorado de ciencias I: marco teórico y formativo, Enseñanza de las Ciencias, v. 28, n. 1, p. 31-46, 2010. 
SHAVIT, P., \& MOSHE, A. The contribution of reflective thinking to the professional development of pre-service teachers. Reflective Practice, v. 20, n. 4, p. 548-561, 2019. https://doi.org/10.1080/14623943.2019.1642190

PEDRO, J. Taking reflection into the real world of teaching. Kappa delta pi record, v. 42, n. 3, p. 129-132, 2006. https://doi.org/10.1080/00228958.2006.10516449

RODRIGUEZ PECH, AMALILLA MOREJON. La complejidad del conocimiento profesional docente y la formación del conocimiento práctico del profesorado. Actualidades Investigativas en Educación, v. 18, n. 2, p. 434-458, 2018. http://dx.doi.org/10.15517/aie.v18i2.33129

SIKES, P. J. Imposed change and the experienced teacher. In: FULLAN, M.; HARGREAVES, A. (Eds.): Teacher development and educational change. Cap. 3, p. 36-55. Routledge, 1992.

VAILLANT, D. Mejorando la formación y el desarrollo profesional docente en Latinoamérica. Revista pensamiento educativo, 41(2), p. 207-222, 2007.

VAN DRIEL, J. H., \& BERRY, A. Teacher professional development focusing on pedagogical content knowledge. Educational researcher, 41(1), p. 26-28, 2012. https://doi.org/10.3102/0013189X11431010

WATTS, M., \& LAWSON, M. Using a meta-analysis activity to make critical reflection explicit in teacher education. Teaching and Teacher Education, 25(5), p. 609-616, 2009. https://doi.org/10.1016/j.tate.2008.11.019

ZEICHNER, K. \& LISTON, P. L. Teaching student teachers to reflect. Harvard Educational Review, v. 57, n. 1, p. 23-48, 1987. https://doi.org/10.17763/haer.57.1.j18v7162275t1w3w

REBER, A. Implicit learning and tacit knowledge. New York: Oxford University Press.

SCHUSTER, A.; PUENTE, M.; ANDRADA, O.; MAIZA, M. La Metodología Cualitativa, Herramienta para Investigar los Fenómenos que Ocurren en el Aula. La Investigación Educativa. Revista Electrónica Iberoamericana de Educación en Ciencias y Tecnología. v. 4, n. 2, p. 109139,2013

WONGSOPAWIRO, D. S. Examining science teachers' pedagogical content knowledge in the context of a professional development program. Leiden University, 2012.

ZEICHNER, K. M. Los profesores como profesionales reflexivos y la democratización de la reforma escolar. In: Volver a pensar la educación (Congreso Internacional de Didáctica), p. 385398, Madrid: Morata, 1995.

\section{NOTAS}

\footnotetext{
i Traducción de la expresión "interconnected model of professional growth" (ICMPG).

ii Para una descripción de la propuesta curricular de referencia véase García y Cutrera (2020).

iii P: promulgación
} 